\title{
Extrahepatic Bile Duct Neuroendocrine Tumor G2
}

National Cancer Institute

\section{Source}

National Cancer Institute. Extrahepatic Bile Duct Neuroendocrine Tumor G2. NCI

Thesaurus. Code C96957.

A well differentiated, intermediate grade tumor with neuroendocrine differentiation that arises from the extrahepatic bile ducts. The mitotic count is 2-20 per 10 HPF and/or the Ki67 index is 3 to 20 percent. 